\title{
Increasing the Research Value of Digitized Fossil Museum Specimens via Integrated Stable Isotope Data
}

Sean Moran ${ }^{\ddagger}$, Bruce MacFadden $^{\ddagger}$, Michelle Barboza ${ }^{\ddagger}$

‡ University of Florida, Gainesville, United States of America

Corresponding author: Bruce MacFadden (bmacfadd@flmnh.ufl.edu)

Received: 10 May 2018| Published: 04 Jul 2018

Citation: Moran S, MacFadden B, Barboza M (2018) Increasing the Research Value of Digitized Fossil Museum Specimens via Integrated Stable Isotope Data. Biodiversity Information Science and Standards 2: e26567. https://doi.org/10.3897/biss.2.26567

\begin{abstract}
Over the past several decades, thousands of stable isotope analyses $(\delta 13 \mathrm{C}, \delta 18 \mathrm{O})$ published in the peer-reviewed literature have advanced understanding of ecology and evolution of fossil mammals in Deep Time. These analyses typically have come from sampling vouchered museum specimens. However, the individual stable isotope data are typically disconnected from the vouchered specimens, and there likewise is no central repository for this information.
\end{abstract}

This paper describes the status, potential, and value of the integration of stable isotope data in museum fossil collections. A pilot study in the Vertebrate Paleontology collection at the Florida Museum of Natural History has repatriated within Specify more than 1,000 legacy stable isotope data (mined from the literature) with the vouchered specimens by using ancillary non Darwin Core (DwC) data fields. As this database grows, we hope to both:

1. validate previous studies that were done using smaller data sets; and

2. ask new questions of the data that can only be addressed with larger, aggregated data sets. 
Additionally, we envision that as the community gains a better understanding of the importance of these kinds of ancillary data to add value to vouchered museum specimens, then workflows, data fields, and protocols can be standardized.

\section{Keywords}

isotope, vertebrate paleontology, fossils, ancillary data, voucher specimens

\section{Presenting author}

\section{Sean Moran}

